\title{
CLASSIFICAÇÃO DA ATIVIDADE ELETROMIOGRÁFICA DO MÚSCULO ORBICULAR DA BOCA EM INDIVÍDUOS COM HANSENÍASE UTILIZANDO A MAQUINA VETORES DE SUPORTE
}

\author{
L. B. Peres*, A. O. Andrade* \\ * Programa de Pós-Graduação em Engenharia Biomédica, Universidade Federal de Uberlândia (UFU). \\ e-mail: lucianolulu2@hotmail.com
}

\begin{abstract}
Resumo: Muitos estudos na área de engenharia biomédica e de ciências da saúde têm buscado a área de aprendizado de máquina para desenvolver métodos que sejam capazes de identificar padrões em diferentes conjuntos de dados. Apesar de extinta em muitos países do primeiro mundo, a hanseníase ainda é uma doença que atinge uma grande parte da população de países como Índia e Brasil. Nesse contexto, esse artigo visa a criação de um método que possibilite futuramente entender como a hanseníase afeta o músculo orbicular da boca utilizando a eletromiografia de superfície e o classificador Máquina Vetores de Suporte.
\end{abstract}

Palavras-chave: Hanseníase, eletromiografia, classificador, Máquina Vetores de Suporte.

\begin{abstract}
A number of studies in the areas of Biomedical Engineering and Health Sciences have employed machine learning tools to develop methods capable of identifying patterns in different sets of data. Despite its extinction in many countries of the developed world, leprosy is still a disease that affects a huge part of the population in countries such as India and Brazil. In this context, this research proposes to develop a method that makes it possible to understand in the future how leprosy affects the muscles of the mouth using electromyography and the classifier support vector machine.
\end{abstract}

Keywords: Leprosy, electromyography, classifier, Support Vector Machine.

\section{Introdução}

Muitos estudos na área de engenharia biomédica e de ciências da saúde têm buscado a área de aprendizado de máquina para desenvolver métodos que sejam capazes de identificar padrões em diferentes conjuntos de dados. Apesar de muitos países declararem que a taxa de incidência da hanseníase diminuíu ao longo dos anos, muitos pesquisadores tem mostrado um cenário diferente. A hanseníase ainda é uma doença que atinge uma grande parte da população de países como Índia e Brasil. Nesse contexto, esse artigo visa a criação de um método que possibilite futuramente entender como a hanseníase afeta o músculo orbicular da boca utilizando a eletromiografia de superfície e o classificador Máquina Vetores de Suporte. Buscou-se primeiro na literatura a forma como pesquisadores ao redor do mundo estão trabalhando com doenças que afetam o sistema nervosa periférico e como a eletromiografia tem atuado para contribuir no entendimento dessas doenças.

A hanseníase é uma doença infecciosa crônica causada pelo Mycobacterium leprae que tem predileção por nervos periféricos e pele [1]. Além de causar alterações dermatológicas, o $M$. leprae nos nervos periféricos provoca desmielinização e posteriormente dano axonal [2]. A bainha de mielina é responsável por acelerar a condução nervosa e quando destruída, provoca perdas na sensibilidade e na motricidade dos músculos inervados por esses neurônios, devido à lentificação da transmissão de informação [2]. Muitos estudos tem chegado a conclusão que a variação dos padrões encontrados em dados de eletromiografia em diferentes doenças tem sido chave para um maior compreendimento da doença. Um dos principais nervos acometidos na hanseníase é o nervo facial [2]. O nervo facial inerva entre outros músculos, o músculo orbicular da boca. Para a avaliação desse músculo foi coletado dados de eletromiografia de superfície (sEMG) em 30 pacientes diagnosticados com hanseníase no Centro de Referência Nacional em Dermatologia Sanitária e Hanseníase (CREDESH) e em 30 indivíduos saudáveis na Universidade Federal de Uberlândia (UFU). Os dados foram analisados e utilizados em um classificador Maquina Vetores de Suporte (SVM) para se identificar quais parametros do classificador são mais relevantes para identificar os padrões gerados pelos dados coletados nos dois grupos.

\section{Materiais e métodos}

Os participantes desse estudo foram divididos em dois grupos. No primeiro grupo (G1) estão os 30 indivíduos saudáveis com idade média de 31,69 anos. Os indivíduos do G1 são divididos em 22 mulheres e 8 homens. O segundo grupo é composto pelos pacientes com hanseníase com média de idade de 35,67 anos. Os indivíduos do G2 são 12 homens e 18 mulheres. Qualquer indivíduo que estivesse utilizando botox ou 
qualquer medicamento que tenha qualquer influência em seu sistema nervoso foi excluído da pesquisa. Também foram excluídos da coleta de dados homens que se recusaram a fazer a barba. A barba aumenta a impedância da pele e isso diminui o nível de sinal/ruído.

Durante a coleta de dados os participantes foram informados sobre o procedimento e todos assinaram o termo de Consentimento Livre e Esclarecido. Esse artigo faz parte do projeto CA A E : 41933614.3.0000.5152 d e no m e Avaliação fonoaudiológica clínica e eletromiográfica da face na hanseníase.

Durante a coleta os pariticpantes foram colocados na posição decúbito dorsal e os eletrodos foram posicionados sobre os músculos estudados. Para este estudo, o músculo escolhido foi o orbicular dos lábios. Como os eletrodos são possuem uma área maior que a dos músculos, eles precisaram ser adaptados como mostra em [3]. Para a coleta dos dados, o pesquisador ficou ao lado do participante fornecendo instruções sobre como deveria ser realizada a coleta dos dados. $\mathrm{O}$ participante deveria realizar o movimento de protrusão labial e manter a contração durante 20 segundos. Após esse tempo, era esperado um tempo de 5 segundos e o processo se repetia mais duas vezes totalizando um total de 3 contrações por participante

Para a coleta de dados foram utilizados eletrodos descartáveis Kendal Meditrace com o sistema Intan de captura de biopotenciais. $\mathrm{O}$ amplificador utilizado foi $\mathrm{o}$ Intan RHD2216.

Os dados coletados foram filtrados com um filtro passa baixa de $500 \mathrm{~Hz}$ e um filtro passa alta de $20 \mathrm{~Hz}$. Esta mesma banda de passagem foi utilizada por [4]. Após a filtragem do sinal foi gerado o sinal de amplitude instantânea e frequência instantânea do sinal bruto. A partir desses sinais pode-se extrair características que representam o dado coletado e não podem ser extraídas do sinal coletado [5].

Após extraídas as características do sinal, foi utilizado um classificador para identificar se é possível reconhecer os padrões gerados pelos dados coletados no G1 e no G2. O classificador utilizado foi o classificador Máquina Vetores de Suporte (SVM). Foi variada a função Kernel do classificador para se identificar qual função apresenta a maior taxa de acerto para os dados utilizados. Dentre os 60 participantes, os dados foram divididos em dois subgrupos de dados. O primeiro subgrupo de dados contendo 20 indivíduos do G1 e 20 do G2 20 foi utilizado para treinamento do classificador. Os outros 10 indivíduos de cada grupo foram colocados no subgrupo de dados que foi utilizado para testes do classificador. Os dados foram separados em cada grupo aleatoriamente através de um algoritmo desenvolvido pelos pesquisadores.

Após os dados terem sido utilizados no classificador, foi feito uma análise estatística quanto a performance do classificador utilizando as funções Kernel linear (KL), radial (KR) e polinomial (KP). A função Kernel é um modelo matemático capaz de através de polinômios traçar um modelo que seja capaz de criar um hiperplano que separe diferentes conjuntos de dados. A análise estatística leva em conta a sensibilidade, especificidade, precisão e acurácia do classificador. Esses dados são resultados de 4 variáveis: Verdadeiros Positivos (VP), Falsos Positivos (FP), Falsos Negativos (FN) e Verdadeiros Negativos (VN). A Tabela 1 mostra como o classificador retorna os dados.

\begin{tabular}{|r|c|c|}
\hline & Previsto & \\
\hline Classe & 1 & 2 \\
\hline 1 & VP & FN \\
\hline 2 & FP & VN \\
\hline
\end{tabular}

Tabela 1: Representação dos dados gerados pelo SVM.

\section{Resultados}

O resultado do classificador pode ser visto na Figura 1. As 4 barras da esquerda representam os resultados do classificador utilizando a função Kernel Linear, as 4 barras centrais representam o resultado utilizando a função Kernel radial e as 4 barras a direita representam o resultado do classificador utilizando a função Kernel polinomial. A Tabela 2 mostra os resultados do classificador utilizando uma função Kernel linear. Nas Tabelas 2, 3 e 4, a Classe 1 representa o G1 e a Classe 2 representa o $\mathrm{G} 2$.

\begin{tabular}{|r|c|c|}
\hline \multicolumn{3}{|c|}{ Kernel Linear } \\
\hline & Previsto & \\
\hline Classe & 1 & 2 \\
\hline 1 & 10 & 0 \\
\hline 2 & 3 & 7 \\
\hline
\end{tabular}

Tabela 2: Resultados do SVM utilizando função Kernel linear.

A Tabela 3 mostra os resultados do classificador utilizando uma função Kernel radial

\begin{tabular}{|r|c|c|}
\hline \multicolumn{3}{|c|}{ Kernel Radial } \\
\hline & Previsto & \\
\hline Classe & 1 & 2 \\
\hline 1 & 10 & 0 \\
\hline 2 & 0 & 10 \\
\hline
\end{tabular}

Tabela 3: Resultados do SVM utilizando a função Kernel radial.

A Tabela 4 mostra os resultados do classificador utilizando uma função Kernel radial

\begin{tabular}{|r|c|l|}
\hline \multicolumn{3}{|c|}{ Kernel Polinomial } \\
\hline & Previsto & \\
\hline Classe & 1 & 2 \\
\hline 1 & 10 & 0 \\
\hline 2 & 2 & 8 \\
\hline
\end{tabular}

Tabela 4: Resultados do SVM utilizando a função Kernel polinomial.

A Figura 1 mostra os resultados do classificador 
utilizando os Kernels linear (KL), radial (KR) e polinomial (KP). As barras na cor azul representam a sensibilidade, as barras na cor marron representam a especificidade, as barras na cor cinza representam a acurácia e a barra amarela representa a precisão.

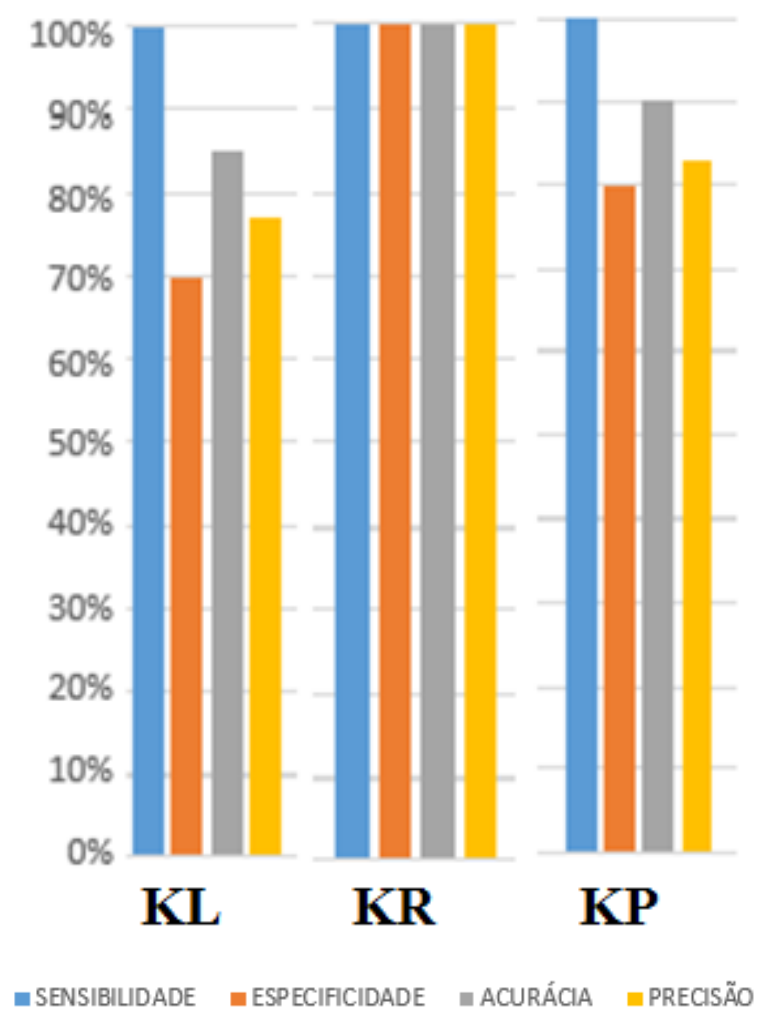

Figura 1: Resultados do classificador SVM utilizando 3 funções Kernel diferentes.

\section{Discussão}

Para os dados utilizados, a função Kernel que apresentou os melhores resultados foi a Radial. $O$ classificador consegui identificar os 10 participantes do G1 como sendo realmente integrantes do G1 e os 10 participantes do G2 como sendo do G2. O músculo orbicular da boca apresentou resultados perfeitos quando utilizado o Kernel radial. Quando se utilizou o Kernel polinomial, o classificador consegiu identificar todos os indivíduos saudáveis porém errou na classificação de apenas dois indivíduos com hanseníase, indicando que apesar de apresentar uma especificidade de $80 \%$ o método é eficiente. Quando se tem um número baixo de participantes, a má classificação de um indivíduo pode levar a uma alta queda de certos parametros do classificador como foi o que aconteceu com a especificidade do Kernel polinomial.

\section{Conclusão}

Quando se procura classificar padrões de dados de eletromiografia em indivíduos com hanseníase e saudáveis, utilizando um classificador SVM, a função Kernel radial apresenta uma taxa de acerto superior do que quando se utiliza uma função Kernel linear e polinomial.

\section{Agradecimentos}

Os autores agradecem aos funcionários do CREDESH, a Dr. Isabela Maria Bernardes Goulard e a doutoranda Marlice Fernandes Oliveira pelo apoio nesse trabalho. Os autores tambem agradecem o apoio financeiro da Fundação de Amparo a Pesquisa do Estado de Minas Gerais (FAPEMIG), do Conselho Nacional de Desenvolvimento Científico e Tecnológico (CNPq) e da Coordenação de Aperfeiçoamento de Pessoal de Nível Superior (CAPES).

\section{Referências}

[1] Rees, RJ. The microbiology of leprosy. In: Hastings, R. C. Leprosy. Medicine in the Tropics Series. Churchill Livingstone. New York, USA. 1985. p. 31-52.

[2] Van Brakel, WH., Nicholls, PG., Wilder-Smith, EP., Das, L., Lockwood, DN. Early Diagnosis of Neuropathy in Leprosy Comparing Diagnostic Tests in a Large Prospective Study (the INFIR Cohort Study). PLoS Negl. Trop. Dis. 2008; 2(4):e212.

[3] Peres, LB., Oliveira, MF., Queiroz CM., Goulart, IMB., Andrade, AO. "Proposta de um protocolo experimental para detecção de atividade eletromiográfica de músculos faciais em indivíduos com hanseníase". In: VIII Simposio de Engenharia Biomédica. 2015.

[4] Boxtel, AV. Optimal bandwidth for the recording of surface EMG activity of facial jaw, oral and neck muscles . Jan. 2015

[5] Andrade, AO. Decomposition and analysis of electromyographic signals. 2005. Tese (Doutorado em Cybernetics. University of Reading, UR, Inglaterra. 